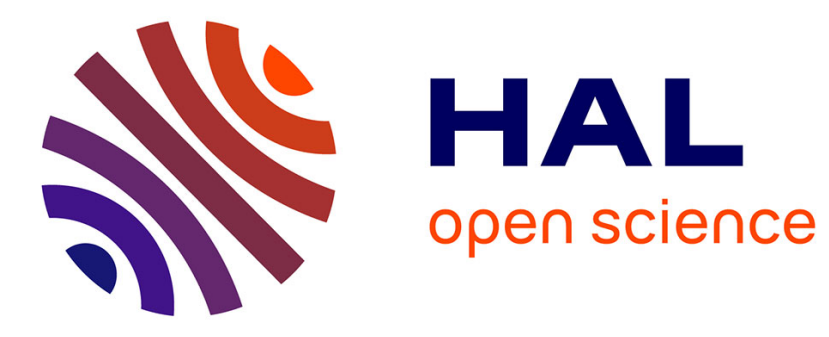

\title{
Un nouveau calendrier de saints égyptien (P. Iand. inv. 318) \\ Jean Gascou
}

\section{To cite this version:}

Jean Gascou. Un nouveau calendrier de saints égyptien (P. Iand. inv. 318). Analecta Bollandiana: revue critique d'hagiographie $=$ A journal of critical hagiography, 1989, 107, 3-4, p. 384-392. halshs00001531

\section{HAL Id: halshs-00001531 https://shs.hal.science/halshs-00001531}

Submitted on 6 May 2004

HAL is a multi-disciplinary open access archive for the deposit and dissemination of scientific research documents, whether they are published or not. The documents may come from teaching and research institutions in France or abroad, or from public or private research centers.
L'archive ouverte pluridisciplinaire HAL, est destinée au dépôt et à la diffusion de documents scientifiques de niveau recherche, publiés ou non, émanant des établissements d'enseignement et de recherche français ou étrangers, des laboratoires publics ou privés. 
Article paru dans:

Analecta Bollandiana, tome 107, fasc.3-4, 1989.

\title{
Jean GASCOU
}

\section{UN NOUVEAU CALENDRIER DE SAINTS ÉGYPTIEN}

\author{
$(P$. Iand, inv. 318 )
}

Les calendriers égyptiens anciens de saints sont très rares ${ }^{1}$. À ce titre, le présent document devrait attirer l'attention des spécialistes du christianisme égyptien.

Sous le titre de Namenliste, vient d'être édité un fragment de papyrus de la collection des Papyri Iandanae, le P. Iand. inv. 318 , de provenance inconnue et attribué au $\mathrm{VI}^{\mathrm{e}}$ ou au $\mathrm{VII}^{\mathrm{e}} \mathrm{s}^{2}$. En voici le texte, emprunté à l'édition princeps, mais où j'incorpore de nouvelles lectures, suggérées par l'examen de la planche:
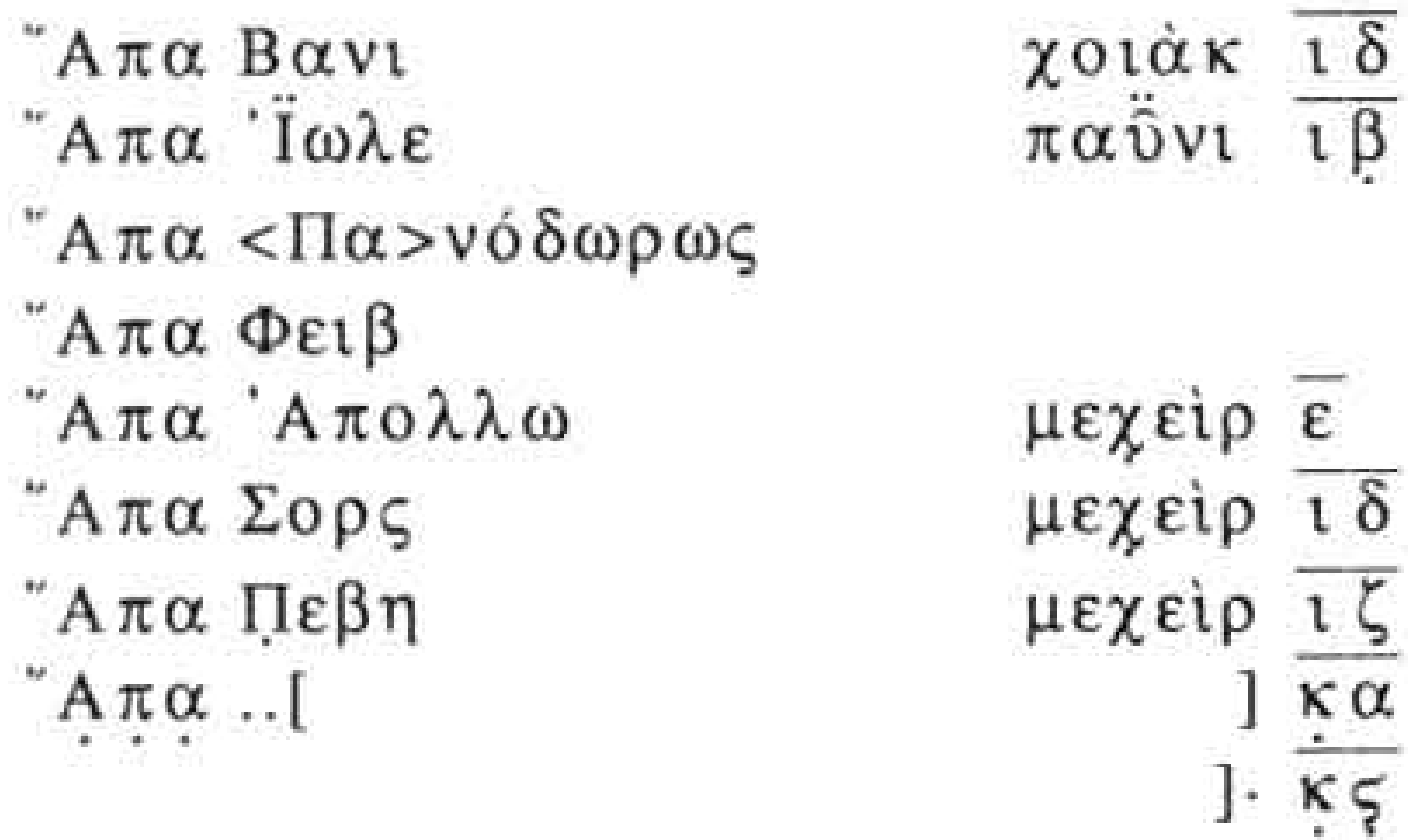

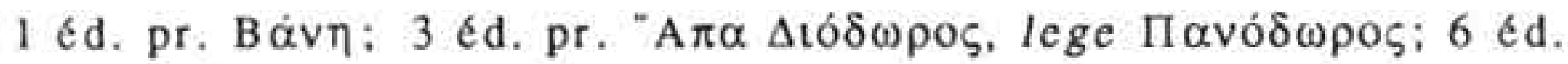
pr. $\mu \varepsilon \kappa \varepsilon i \rho$

La Liste des abréviations figure en fin d'article, p. 392.

1 En grec, le célèbre $P$. Oxy. XI 1357 éd. et comm. par le P. Delehaye dans Anal. Boll. 42 (1924), p. 83-99 (voir VAN HAELST, n 961). On trouvera aussi quelques éléments pour les mois de pachôn, pauni et epeiph dans un document comptable du début du $\mathrm{VI}^{\mathrm{e}} \mathrm{s}$. provenant d'Aphroditô, P. Cairo Masp. II 67141, $\mathrm{f}^{\circ} \mathrm{V}, r, 21-30$. En copte, je n'ai pu noter que l'ostracon CO 26 (voir 27), venant de Haute-Égypte, les inscriptions de Baouît, p. 5-6, Saqqara, p. 69-70, $n^{\circ} 226$, Esna I, p. 104, n ${ }^{\circ} 67$, a vec le comm. de R. G. Coquin, Esna IV, p. 49-52, et le ms. sur parchemin attribué au $\mathrm{V}^{\mathrm{e}}-\mathrm{VI}^{\mathrm{e}}$ s., provenant peut-être d'Oxyrhynque, qui a été publié par CRUM, Calendar. J'aurais pu augmenter cette liste de tel ou tel papyrus ou ostracon donnant le jour de fête de tel saint, mais il ne s'agit plus de calendriers. Au total, donc, fort peu de choses, et la remarque faite par le P. Delehaye dans Les martyrs d'Égypte (Anal. Boll., t. 40 [1922], p. 91, n. 1) sur la rareté des calendriers égyptiens me paraît toujours actuelle.

2 P. J. SIJPESTEIJN et K. A. WORP, Einige Papyri aus den Giessener Papyrussammlungen, dans Aegyptus 67 (1987), p. 69-70, $\mathrm{n}^{\circ} 61 \mathrm{et}$ pl. XII. L'écriture, une capitale très grossière, ne permet pas d'être plus précis sur la datation. 
Je crois pouvoir définir cette liste comme un calendrier de saints, notamment, comme on le verra, monastiques. Il ne s'agit pas seulement de la disposition, noms de personnes et jours de mois, du titre d' $\alpha$ $\pi \alpha$, porté par chacun de ces personnages ${ }^{3}$. Notre argument principal se tire en fait de la 1. 5: «Apa Apollô, le 5 mecheir».

Rapprochons en effet ce passage de $S B \times 10269$, texte de

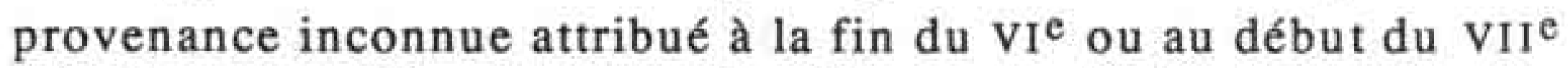
s. ${ }^{4}$. Dans cette lettre au grec fautif, un certain Andreas annonce à Ammônios (un moine, visiblement) que (1. 5-7): $\dot{\varepsilon} \lambda \pi \hat{i} \sigma \omega$ عí

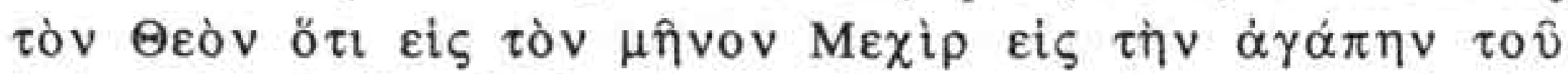

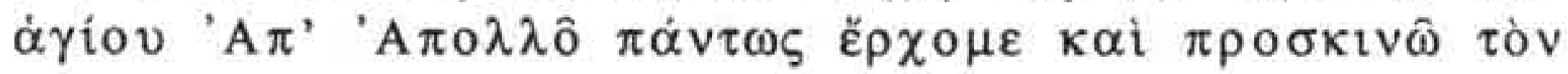
$\dot{v} \mu \omega \hat{v} \pi \alpha \tau \varepsilon \dot{\rho} \alpha \nu$. On pourrait traduire: «J'espère, m'en remettant à Dieu, que, au mois de mechir, je viendrai sans faute, à l'agapè 5 du saint Apa Apollô, présenter mes respects à notre Père» ( $\dot{v} \mu \hat{\omega} v$ pour $\dot{\eta} \mu \hat{\omega} \mathrm{v}$ ).

De toute évidence, nos deux textes se rapportent au même saint Apa Apollô et à sa commémoraison de mecheir que notre P. Iand. inv. $318,1.5$, permet de fixer plus précisément au 5 de ce mois, soit au 30/31 janvier. Par analogie, on en déduira que les autres dates du document correspondent à autant d' $\dot{\alpha} \gamma \alpha \dot{\alpha} \pi \alpha_{l}$ d'autres saints.

On établit facilement qui est cet Apollô. Son nom, dans $P$. Iand. inv. 318, est immédiatement précédé, 1. 4, par celui d'Apa Pheib, mais sans indication de date. Pheib et Apollô se fêtaient donc le même jour, soit le 5 mecheir $^{6}$. Cela suggère fortement que nous avons affaire à l'A pollô de Bawît et à son ami Phib, si souvent associés dans la littérature hagiographique, les inscrip-

${ }^{3}$ Rappelons que le titre d'apa ne marque pas à coup sûr l'appartenance à un milieu religieux (cf. $P$. Vindob. Salomons 10, n. 3: $P$. Hamb. III 228, n. 13).

${ }^{4}$ P. Yale inv. 1773 (cf. Liste des abrév.).

5 «Feast», édition princeps; YOUTIE, p. 262-263 note qu'il s'agit plus précisément d'un banquet funéraire commémoratif (généralement associé à une célébration eucharistique; voir E. F. BRUCK, Totenteil und Seelgerăt im griechischen Recht, Munich, 1926, p. 289-290).

${ }^{6}$ Ainsi qu'un mystérieux Panodôros (voir ci-dessous, p. 390). 
tions et l'iconographie ${ }^{7}$. Peut-être le monastère de $S B \times 10269$ n'est-il autre que celui de Bawît ${ }^{8}$.

Cette date du 5 mecheir pour l' $\dot{\alpha} \gamma \dot{\alpha} \pi \eta$ d'Apollon et Phib, confrontée aux données des sources médiévales, pose de difficiles problèmes. Voici l'état de la question, d'après une étude récente de R.-G. Coquin dont je me sens largement tributaire9.

Selon le synaxaire de Basse-Égypte ${ }^{10}$, la fête d'Apollô, adjoint à Phib, "suivant la coutume de la Haute-Égypte", se plaçait le 25 bâbeh ( 25 phaôphi; 22/23 octobre), usage confirmé, pour la Haute-Égypte, par les غ́ $\mu \eta \eta \varepsilon \hat{\imath} \alpha \iota$ du Couvent Blanc ${ }^{11}$. De même, le calendrier d'Abû 1 -Barakât ${ }^{12}$ et les ménologes ${ }^{13}$ mentionnent respectivement pour le 25 bâbeh "Apollon, moine de Thébaïde" et "Abba Apollon le moine”, encore que sans allusion à $\mathrm{Phib}^{14}$.

7 Voir CoQuin, Apollon; ID., Esna IV, p. 56-57 et 68-69. Précisons que le nom de l'éponyme de Bawît est Apollô ou Apollôs et non pas Apollon.

8 Hypothèse avancée par YOUTIE, p. 263-264, qui n'écarte pas, pourtant, un monastère homonyme d'Aphroditô dont le patron n'a rien à voir avec notre Apollô. Pour la documentation papyrologique de Bawit, voir M. KRAUSE et KI. WESSEL, $R B K \mathrm{I}$, col. 571. Sur le monastère, voir en dernier lieu TIMM, CKÄ, II, p. 643-653. Nous disposons d'archives grecques et coptes provenant d'un monastère hermopolite d'Apa Apollôs, du village de Titkôis/Titkooḥ. COQUIN, Apollon, p. 435.438, le distingue de celui de Bawît, ce que paraît contester J. R. REA, Journal of Egyptian Archaelogy, 71 (1985), Reviews Supplement, p. 70. Pour le dossier, voir H. HARRAUER et P. J. SIJPESTEIJN, Ein Darlehen von Wein, dans Chronique d'Égypte, 57 (1982), p. 296-302 [à propos de l'actuel $S B$ XVI 12401; cf. K. A. WORP. P. Vindob. Barbara inv. 266, dans Chronique d'Égypte, 59 (1984), p. 145-148], ainsi qu'un document copte de Milan publié par S. PERNIGOTTI dans Aegyptus, 65 (1985), p. 101-105.

9 COQUIN, Apollon.

10 Éd. R. BASSET, PO I, p. 366.

11 PLEYTE \& BOESER, p. 216, et WESSELY, SPP XVIII, 1917, p. 7, $n^{\circ}$ 263a. COQUIN, Apollon, p. 443, se réfère aussi au feuillet inédit de Vienne K 9736. Pour cette documentation, voir en dernier lieu QUECKE, Zwei Blatter.

12 Éd. E. TISSERANT, POX, p. 257.

13 Éd. F. NAU, PO X, p. 190.

14 Toutefois, le ms. F de l'éd. Nau présente une variante au nom d'anba Abîb (Phib), signalée dans $P O X$, p. 190, n. 7 (voir p. 224). 
Pour le 5 mecheir, les $\dot{\rho} \mu \eta v \varepsilon i ̂ \alpha t$ du Couvent Blanc n'évoquent qu'Apa Pšoï ${ }^{15}$. Rien dans le synaxaire de Haute-Égypte ${ }^{16}$. Quant aux sources concernant la Basse-Égypte: le calendrier de Saint-Jérémie de Saqqara mentionne, très probablement pour un 5 mecheir d'après le contexte, un Apollô indéterminé ${ }^{17}$. Le calendrier d'Abû l-Barakât et deux mss. des ménologes parlent bien d'un Apollon, mais il s'agit d'Apollon le solitaire, identifiable à Apollon le berger, moine de Scété, de Basse-Égypte donc ${ }^{18}$, et dont la Vie arabe fixe en effet la fête au 5 amšîr ${ }^{19}$.

Cependant, à cette date, la notice du synaxaire de BasseÉgypte porte: «En ce jour, mourut aussi saint Anbâ Apollo (Balou) qui ressemblait aux anges [épithète consacrée d'Apollô de Bawît]. Son histoire est écrite au 25 de bâbeh" 20 .

Selon Coquin, le rédacteur du synaxaire a vraisemblablement confondu les deux saints, Apollô de Bawît et Apollon le berger $^{21}$.

On ne peut plus dire, au vu de notre calendrier, que le rédacteur du synaxaire de Basse-Égypte ait commis une confusion. Il enregistre correctement le fait qu'à une époque relativement ancienne du christianisme égyptien, à tout le moins en certains lieux 22 , Apollô de Bawîț et même Phib se fêtaient bien le 5 mecheir, mais qu'il y eut plus tard, à partir de la Haute-Égypte,

15 PLEYTE \& BOESER, p. 195, et WESSELY, SPP XVIII, 1917, p. 10 , $\mathrm{n}^{\circ} 264$ b. Voir COQUIN, Apollon, p. 444, n. 44.

16 Inédit, mais voir COQUIN, Apollon, p. 444, Sur cette recension, voir du même auteur, Le synaxaire des Coptes, dans Anal. Boll. 96 (1978), p. 351-365.

17 Saqqara, p. $69, \mathrm{n}^{\circ} 226,17$. Il doit s'agir de l'Apollô de Bawît (cf. p. $71, n .6$ ). On sait que le monastère de Saint-Jérémie de Saqqara est influencé par Bawît.

18 POX, p. 264 ; mss. E et H de l'éd. NAU, ibid., p. 199, n. 5 et p. 213 et 227. Par ailleurs, le nom d'Apollô, au lieu de Paul, est rétabli dans le ms. A de l'éd. NAU, ibid., p. 199, par U. ZANETTI, Les lectionnaires coptes annuels : Basse-Égypte, (= Publications de I'Institut Orientaliste de Louvain, 33), Louvain-la-Neuve, 1985, p. 107 . Pour les sources concernant l'Apollon de Scété, voir COQUIN, Apollon, p. 444 et nn. 47 et 48 .

19 D'après des mss. inédits auxquels se réfère COQUIN, Apollon, p. 444, n. 49.

20 PO XI, p. 793.

21 COQUIN, Apollon, p. 444.

22 On ne saurait trop déplorer, sur ce point, l'incertitude pesant sur les provenances respectives de $P$. Iand. inv. 318 et de $S B \mathrm{X}$ 10269. 
déplacement du souvenir d'Apollô au 25 phaôphi qui, en principe, était seulement une fête funéraire de Phib.

La raison s'en perçoit assez bien. La fête de phaôphi, comme l'a établi Coquin, comportait un rite d'inclination pénitentielle, une "petite $\mu \varepsilon \tau \alpha \dot{\alpha} o t \alpha$ " assurant la rémission des péchés, qui la rendit vite et partout populaire: dès 535/536 par exemple, nous voyons "le jour de la metanoia" célébré le 25 phaôphi à Oxyrhynque, cité qui n'appartenait pas, historiquement, à la Haute-Égypte (l'ancienne province de Thébaïde) ${ }^{23}$. D'autre part, la petite metanoia passait pour avoir été instituée par Apollô en mémoire de son ami. En ce jour exceptionnel du 25 phaôphi, on ne pouvait manquer d'unir Phib et Apollô dans la même commémoraison. La fête du 5 mecheir, d'un caractère sans doute plus banal, dut souffrir de cette concurrence. L'affaire aurait pu, à la longue, tourner à l'avantage exclusif de Phib, "l'homme de la pénitence salutaire"24. C'est un fait pourtant que, dans le calendrier d'Abû 1-Barakât et dans les ménologes, le prestigieux éponyme de Bawît, à la date du 25 bâbeh, a totalement éliminé Phib ${ }^{25}$.

Pour expliquer l'intrusion d'A pollon le berger, à la date du 5 mecheir, dans le calendrier d'Abû l-Barakât et dans certains mss. des ménologes, on ne peut guère former qu'une hypothèse.

Il se peut que les milieux chrétiens de Basse-Égypte, une fois reçue chez eux la double fête du 25 phaôphi, au lieu d'expurger le nom d'Apollô de Bawît de leurs calendriers à la date du 5 mecheir - comme ce fut probablement le cas en Haute-Égypte - aient préféré en altérer l'identité, en prenant appui sur les traditions locales concernant un Apollon de Scété.

23 Voir d'une manière générale COQUIN, Apollon, p. 438-446 et, plus spécialement, p. $442-443$ sur le calendrier d'Oxyrhynque $P$. Oxy. XI 1357 et son jour de la metanoia (1. 4; cf. n. 1 ci-dessus).

24 Baouît, p. 120.

25 Aussi dans le ms. viennois inédit K 9736 appartenant au dossier des hermèneiai du Couvent Blanc, qui ne parle plus, à cette date, que de "la prostration d'Apollô" (cf. COQUIN, Apollon, p. 443). Noter toutefois, à propos des ménologes, l'exception signalée à la n. 14 cidessus. Encore que nous disposions d'une hagiographie copte et arabe de Phib (ce qui n'est plus le cas pour Apollô), la personnalité d'Apollô y domine (ibid., p. 435), en tant, notamment, qu'instaurateur de la fête du 25 phaôphi et de son rite singulier. 
Pour faciliter la réinterprétation on aura, au besoin, changé dans dans sa biographie la date de sa mort ${ }^{26}$.

Je passe à présent en revue les autres saints énumérés par $P$. Iand. inv. 318, en précisant que je dois beaucoup au concours désintéressé de M. Pezin. Que d'incertitudes pourtant!

- ${ }^{\prime A} \pi \alpha \mathrm{B} \alpha v \imath, 14$ choiak (10/11 décembre), 1. 1.

Sans doute faut-il, avec M. Pezin, voir dans le nom Bani une variante dialectale (fayoumique, bohaïrique ou sahidique ${ }^{f}$ ) du

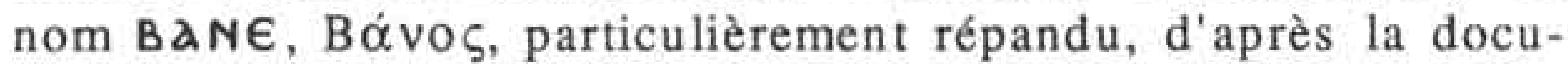
mentation papyrologique grecque et copte, dans la région d'Ašmûnayn/Hermopolis Magna27.

Dans l'Hermopolite (et donc non loin de Bawîț), vécut précisément, à la fin du IV e $s$. et au début du ve, l'ascète $a \Pi a$ BaNE. Son souvenir reste attaché à l'actuel Dayr Abû Fânâ, dans le désert à l'ouest de Hûr. Pour son dossier, on se reportera en dernier lieu à TIMM, CKÄ II, p. 573-574. Voir aussi Bibliotheca Orientalis, 43 (1986), col. $97^{28}$.

Une des Vies arabes de Bane, contenue dans le ms. Par. ar. 153 , f. $216^{\mathrm{r}}-225^{\mathrm{r}}$, fixe sa fête au 25 amšîr (19/20 février), ce qui ne $s^{\prime}$ accorde pas avec notre texte ${ }^{29}$.

- "A $\pi \alpha{ }^{\prime} I \omega \lambda \varepsilon, 12$ pauni (6 juin), 1. 2.

Le nom Iôle peut correspondre à $\operatorname{lor} \lambda \epsilon / \operatorname{lor} \lambda l /$ 'Iov́ $\lambda$ tos. Le célèbre hagiographe oxyrhynchite Jules d'Aqfahs (Kbehs) paraît exclu ici, car sa fête, d'après le synaxaire de BasseÉgypte, tombe un 22 tout (thôth; $19 / 20$ septembre) ${ }^{30}$.

Un candidat plus probable à l'identification serait le moine héracléopolite ana lor $\lambda \mathrm{I}$, martyrisé en compagnie d'Apa

26 Dans ce retraitement, la seconde recension du synaxaire éthiopien pourrait marquer une étape: à la date du 5 yakâtît on y trouve en effet, après une courte mention d'Apollô "semblable aux anges" empruntée au synaxaire de Basse-Égypte, une "assez longue notice sur Apollon le berger» (COQUIN, Apollon, p. 444, n. 50).

$27 \mathrm{M}$. Pezin rejette, pour ce nom, l'étymologic d'après le nom copte du dattier (voir H. MUNIER, Les monuments coptes, dans Bulletin de la Societé d'Archéologie Copte, 6 [1940], p. 148, n. 2), me faisant remarquer que le dattier se dit $B N N \epsilon^{s}, B \in N I^{f}, b$, et jamais $B A N \in$. Il suggère un rapprochement avec $B \omega \omega N^{5}$ et BANIf, "mauvais".

28 C.R. par J. GASCOU de J. M. DIETharT, Corpus Papyrorum Raineri IX, Griechische Texte VI, Vienne, 1984.

29 Renseignement communiqué par M. Pezin.

30 PO I, p. 290. 
Ptolémée un 21 tôbe (tubi; $16 / 17$ janvier) ${ }^{31}$. On remarquera en effet que cette date, à la différence du présent 12 pauni, s'intercalerait au mieux, dans notre calendrier, entre le 14 choiak de la 1. 1 et le 5 mecheir de la 1. 5. On pourrait expliquer l'anomalie chronologique du 12 pauni comme suit: à une date plus ancienne, les milieux religieux d'où émane $P$. Ianda. inv. 318 célébraient bien la fête d'Apa Iôle en tubi. Pour telle ou telle raison, on changea le jour de sa fête, mais on négligea de transférer Iôle sur les listes de pauni, et on se contenta de remplacer le 21 tubi par le 12 pauni $^{32}$.

Contre ces hypothèses, on peut cependant faire valoir l'absence, dans notre papyrus, du compagnon de martyre de Iouli, l'A pa Ptolémée.

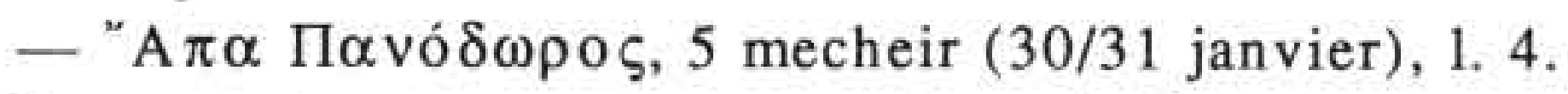

Le nom de ce personnage est restitué en supposant une haplographie, mais à ma connaissance, ni la littérature hagiographique égyptienne, ni les papyrus, ni les inscriptions n'enregistrent un saint Panodôros.

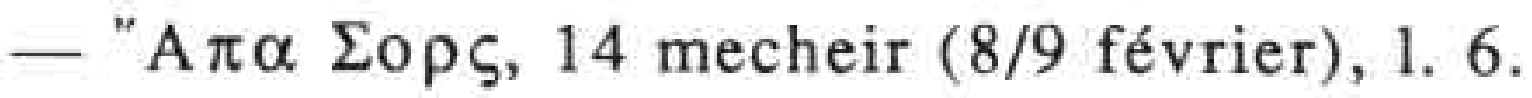

On pourrait voir dans le noms $\Sigma o \rho \varsigma$ une corruption de

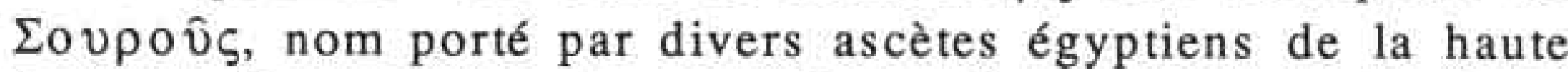

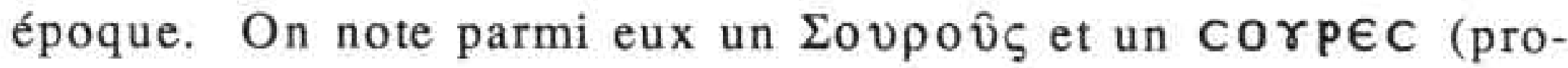
bablement identiques), liés à Anoup et Apollô de Bawît ${ }^{33}$. Mais nous ignorons la date de leur commémoraison.

$\Sigma o \rho \varsigma$ peut encore représenter $\Sigma \varepsilon v \hat{\eta} \rho \circ \varsigma$ ou $\Sigma \varepsilon \circ v \hat{n} \rho \circ \zeta$. Or le 14 mecheir, selon les calendriers médiévaux, dont les $\dot{\rho} \rho \mu \eta$ veî $\iota_{1}$ du Couvent Blanc ${ }^{34}$ et le synaxaire de Basse-Égypte,

31 Voir E. A. E. REYMOND et J. W. B. BARNS, Four Martyrdoms from the Pierpont Morgan Coptic Codices..., Oxford, 1973, p. 136.

32 Le 21 tubi, l'Église Copte célèbre la «mort de la Vierge Marie» (dont le corps mort a été transporté au ciel le 22 mesôre). Une com. munication récente du P. M. van Esbroeck (La Tradition copte de la fête de la dormition de la Vierge: IVe Congrès International d'Études Coptes, Louvain-la-Neuve, 1988 - à paraître dans les Actes) soutient que cette fête du 21 tubi remonte aux luttes qui ont suivi le concile de Chalcédoine: le rapprochement de l'hypothèse dont il est question cidessus avec la date avancée par $M$. van Esbroeck pourrait être fructueux. Par ailleurs, il convient de noter que le 12 pauni est, dans tous les calendriers liturgiques, la fête de l'archange S. Michel; il est évident qu'elle ne l'était pas encore à l'époque de ce papyrus, dans la mesure où la lecture est exacte. [N.D.L.R.]

33 Cf. R. G. COQUIN, Esna IV, p. 72-73.

34 PLEYTE et BOESER, p. 198. 
marque la fête de Sévère d'Antioche ${ }^{35}$. L'illustre docteur étant mort en $538^{36}$, nous aurions ainsi un terminus post quem de notre texte.

- "А $\pi \alpha$ Пєßク, 17 mecheir (11/12 février), 1. 7.

Le nom de $\Pi \varepsilon \beta \varepsilon ́$ ou $\Pi \varepsilon \beta \hat{\eta} \varsigma$ était déjà bien attesté par les papyrus. Dans P. Lond. VI 1917, 11, 17, 21 et 26 (?), docu-

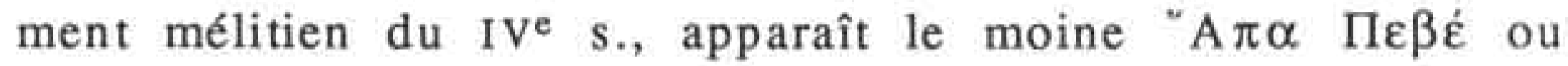

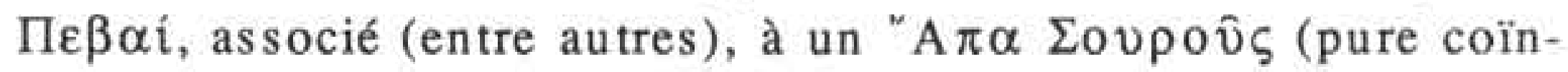
cidence naturellement). Pour le reste, la personnalité de ce saint m'échappe.

Strasbourg-II

Jean GASCOU

Summary. Papyrus Iandana inv. 318 previously published as a "Namenliste" (Aegyptus, 67 [1987], p. 69-70) is in fact a calendar of Coptic saints. Among other points of interest, it vindicates the accuracy of the entry of Synaxar, 5 amšîr, devoted to Saint Apollos.

35 PO XI, p. 823-825.

36 E. STEIN, Histoire du Bas-Empire II, Paris (etc.), 1949, p. 384 (qui signale que 1a date de 539 serait aussi possible: ibid., n. 3). 


\section{LISTE DES ABRÉVIATIONS}

Baouît $=\mathrm{J}$. CLÉDAT, Le monastère et la nécropole de Baouît, (= Mémoires publiés par les membres de l'IFAO, XII), Le Caire, 1904.

$C O=$ W. E. CRUM, Coptic Ostraca from the Collection of the Egypt Exploration Fund, the Cairo Museum and others, Londres, 1902.

COQUIN, Apollon = R.-G. COQUIN, Apollon de Titkooh ou/et Apollon de Bawît?, dans Orientalia 46 (1977), p. 435-446.

CRUM, Calendar $=$ W. E. CRUM, Fragments of a Church Calendar, dans Zeitschrift für die Neutestamentliche Wissenschaft, 37 (1938), p. 23-32 (cf. le CR du P. PEETERS dans Anal. Boll., 61 [1943], p. 271-273).

Esna $=\mathrm{S}$. SAUNERON et al., Les ermitages chrétiens du désert d'Esna, (= Fouilles de I'IFAO, XXIX), 4 vol., Le Caire, 1972.

P. Cairo Masp. = J. MASPERO, Papyrus grecs d'époque byzantine, (Serv. des Antiq. de l'Égypte, Cat. gén. des Antiquités égyptiennes

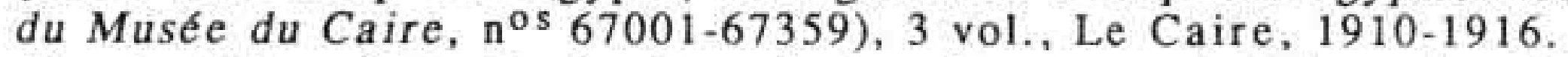

P. Hamb. III = Griechische Papyri der Staats- und Universitatsbibliothek Hamburg, herausg. von B. KRAMER und D. HAGEDORN (= Papyrologische Texte und Abhandlungen, XXXI), Bonn, 1984.

P. Lond. = Greek Papyri in the British Museum, Londres, 1893- .

P. Oxy. = The Oxyrhynchus Papyri, Londres, 1898-.

$P$ Vindob. Salomons $=$ R. P. SALOMONS, Einige Wiener Papyri, Amsterdam, 1976.

P. Yale inv. $1773=$ éd. pr. D. SAMUEL, A Byzantine Letter in the Yale Collection, dans Bulletin of the American Society of Papyrologists, 4 (1967), p. 37-42; voir la réédition par H. C. YouTIE, dans Zeitschrift für Papyrologie und Epigraphik, 16 (1975), p. 259-264 $(=$ Scriptiunculae posteriores, I, Bonn, 1981, p. 163-168) $=S B \mathrm{X}$ 10269.

PLEYTE et BOESER = W. PLEYTE et P. A. A. BOESER, Manuscrits coptes du Musée d'Antiquités des Pays-Bas à Leide, Leiden, 1897.

$P O=$ Patrologia Orientalis, Paris puis Turnhout, 1903- .

QUECKE, Zwei Blätter $=\mathrm{H}$. QUECKE, Zwei Blätter aus koptischen Hermeneia-Typika in der Papyrussammlung der Österreichischen Nationalbibliothek (P. Vindob. K 9725 und 9734), in Festschrift zum 100jährigen Bestehen der Papyrussammlung der Österreichischen Nationalbibliothek (Vienne, 1983), p. 194-206 et pl. 10 et 11.

$R B K=$ Reallexikon zur byzantinischen Kunst, Stuttgart, 1966- .

Saqqara = J. E. QUIBELL, Excavations at Saqqara (1908-9, 1909-10): The Monastery of Apa Jeremias, Le Caire (Service des Antiquités de l'Égypte), 1912.

$S B=$ Sammelbuch griechischer Urkunden aus Ägypten, Strasbourg puis Heidelberg puis Wiesbaden, 1915.

$S P P=$ Studien zur Palaeographie und Papyruskunde, herausgegeben von $D^{r}$ Carl WESSELY, 23 vol., Leipzig, 1901-1924.

TIMM, CKÄ = S. TIMM, Das christlich-koptische Ägypten in arabischer Zeit. Sammlung ăgyptischer Toponyme, = Tübinger Atlas des Vorderen Orients (TAVO), Beihefte, B, 41, Wiesbaden, 1984 ss.

VAN HAELST $=\mathrm{J}$. VAN HAELST, Catalogue des papyrus littéraires juifs et chrétiens, Paris, 1976

YOUTIE $=$ H. C. YOUTIE, Zeitschrift für Papyrologie und Epigraphik, 16 (1975), p. $259-264$ (= Scriptiunculae posteriores, I, Bonn, 1981, p. 163-168). 